\title{
The Characteristic in Infinite Dimension Space
}

\author{
Qing Li 10 \\ Shijiazhuang Traditional Chinese Medical Hospital, Shijiazhuang, China \\ Email: liqingliyang@126.com,2958773632@qq.com
}

How to cite this paper: $\mathrm{Li}, \mathrm{Q}$. (2021) The Characteristic in Infinite Dimension Space. Advances in Pure Mathematics, 11, 645-651. https://doi.org/10.4236/apm.2021.117042

Received: May 26, 2021

Accepted: July 2, 2021

Published: July 5, 2021

Copyright (C) 2021 by author(s) and Scientific Research Publishing Inc. This work is licensed under the Creative Commons Attribution International License (CC BY 4.0).

http://creativecommons.org/licenses/by/4.0/

\begin{abstract}
In modern mathematics, geometric and algebraic properties of space can be applied by calculus in which the concept of gradually increasings of dimensions is existence (such as zero-dimension, one-dimension, two-dimension, and three-dimension, etc). However, this is not fact because some new concepts have been put forward in this paper where there is only a concept of infinitely great that is one quantitative continuum implied by the change of direction. The accurate description of this one quantitative continuum is that its parts are connected each other as a unity at the infinite distance (infinitely great) relative to any orientation (all orientations) of our existence. It is unity in which its random parts are these infinitely great quantities and thus we call this unity as infinite quantities of infinite dimensions.
\end{abstract}

\section{Keywords}

Infinite Dimension, The Change of Direction, One Quantitative Continuum

\section{Introduction}

The dimension of space is the core of modern mathematics. In modern mathematics, the parts of space are characterized by points (singularity), lines, planes, surfaces of higher dimensions, etc., which also correspond to zero-, one-, two-, higher-dimensions, and so on. In order to study the geometric and algebraic properties of space, many modern mathematical tools and branches emerged, such as differential geometry, algebraic geometry, etc., the core of which is calculus. For example, differential geometry is a branch of mathematics that uses the theory of calculus to study the geometric properties of space. Classical differential geometry studied curves and surfaces in three-dimensional space, while modern differential geometry began to study more general space-manifolds.

There are, however, a few problems. For example, for any given curve segment, the infinitesimal division of calculus can only be used to calculate its approximate length, and this approximate length obtained is also compared with 
other different measures of length. Whatever new mathematical methods and tools we use, the exact length of this segment may never be calculated because it involves the problem of getting infinitesimal precision. Because of Axioms 1 and 3 ref. [1] [2], the exact value of this line segment can now be obtained. It is unimaginable that in Axiom 3, infinitesimal doesn't exist, and the continuum space we see is only infinitely great that is one-quantity. The specific discussion about them is in my other papers.

In this paper, the characteristics of dimension of space based on Axiom 3 will be discussed. Interestingly, being different from the concept of gradually increasings of dimension in modern mathematics, such as zero-dimension, one-dimension, two-dimension, and three-dimension, and so on, there is only a concept of infinitely great that is an one quantitative continuum in Axiom 3, in which its parts are connected each other as a unity at the infinite distance (infinitely great), and we call this unity as infinite quantities of infinite dimensions.

\section{Main Results}

Preliminaries 1 . It is vague concept in which the dimension of space in common sense is described as 0 dimension, one-dimension, two-dimension, and threedimension, etc., corresponding to undividedly 0 point, the straight line, plane surface, and curved surface, etc. To define the concept of dimension more clearly, I assume that above definition of dimension is correct and its implications must be illustrated in Axiom1 and Axiom 2 ref. [1]. In Axiom 1 the change in dimension is the change among finite quantities, such as change from 0 to onedimension, or from one-dimension to two-dimension. In Axiom 2, 0 dimension (zero-dimension) is non-existent and change from one-dimension to two-dimension is the alterations from finite quantities to infinite quantities. Supposing one-dimension is 1 , then two-dimension is $\infty$ (infinitely great), the formula for $\infty / 1=\infty$ is established. The more high dimension is described as $\infty^{n}(n \geq 3$, until $\mathrm{n}=\infty)$. In view of the common feature of the two axioms is to be able to compare sizes, and the superpositions of numerical quantities in the two axioms is gradually close to an given length quantities (or numerical quantities is gradually increase), the numerical quantities of dimension in the two axioms is aslo gradually increase (namely one-dimension, two-dimension, and three-dimension, the more high dimension, until infinite dimension) in spite of their different definitions of dimension.

Preliminaries 2. Since Axiom 1 and Axiom 2 are logically incorrect, the above definition of dimension does not exist. From revised Axiom 1 (in this article I call revised Axiom 1 as Axiom 3) the exact definition and some properties of dimension are obtained ref. [2].

Theorem 1. In Axiom 3 the infinitesimal is defined as one-dimension quantities of finite length “-” without a size. The exact definition for this one-dimension “-” means: 1) A straight line of finite length. 2) Without a size. 3) Onedimension absoluteness. That is, in terms of extensibility of space, it is a straight 
line in one-dimension, while in other orientations, it is 0 dimension. Seeing Figure 1.

Theorem 2. In Axiom 3 the finite quantities attain to the accumulations of infinitely many quantities of itself by the way of change of direction, reaching infinitely great. This infinitely great (infinitely many quantities) indicated by change of direction exists in the form of one quantitative continuum. The infinitely great can be described as unlimited, open space, compressing anything outside it into nothing. The change of direction indicated that the finite quantities is not part of infinite quantities and all the parts of space we can see are this one quantitative continuum that represents infinitely great, and in which you can not compare sizes of each other and carried out by the any algorithms, such as the operations of addition, subtraction, multiplication, division. Since without position of finite quantities, accurate description of this one quantitative continuum is that its parts are connected each other as a unity at the infinite distance (infinitely great) relative to any orientation (all orientations or all azimuth) of our existence. Here any orientation (all orientations) of our existence is indicated accurately as infinity of space whose parts are connected each other as a unity at the infinite distance (open, unlimited space). For a clearer description, the terms "its parts" is used in this article, in fact the concept of "its parts" is equal to one quantity. Seeing Figure 2.

Consequently, Axiom 3 is denial of feature of gradually increasings of number and dimension quantities. In Axiom 3, there is no concept of 0 dimension, onedimension, two-dimension, and three-dimension, the more high dimension, until infinite dimension, namely the concept of gradually increasings of dimension is non-existence. On the other hand, there is only a concept of infinitely great that is an one quantitative continuum and is not established by the any algorithms within it.

A conclusion is drawn that the quantitative values and dimensions are the same thing, it is unity in which its random parts is this infinitely great quantities. Thus we call this unity as infinite quantities of infinite dimensions. Therefore, the space we see is just the difference of one-dimension finite quantities and infinitely great quantities that is open space and is implied by change of direction

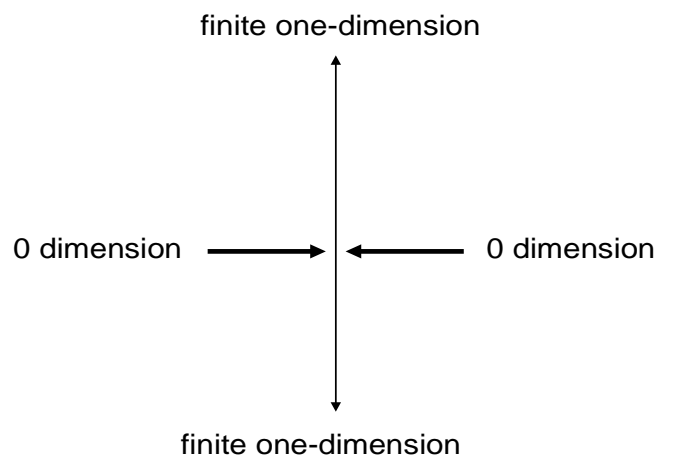

Figure 1. A straight line of finite length. Without a size. In terms of extensibility of space, it is a straight line in one-dimension, while in other orientations, it is 0 dimension. 


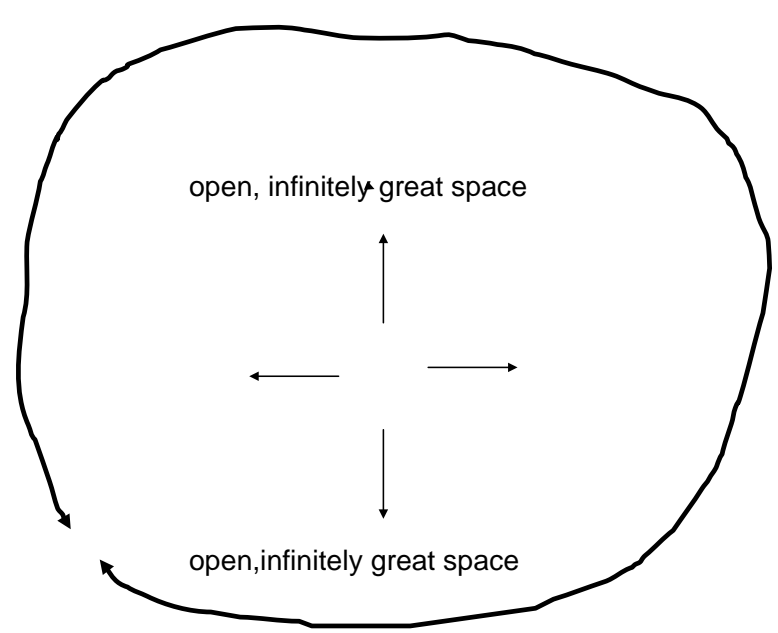

All parts are made of infinite quantities, connect each other in infinitely great distance

Figure 2. one quantitative continuum is that its parts are connected each other as a unity at the infinite distance (infinitely great) relative to any orientation (all orientations) of our existence. Here any orientation (all orientations) of our existence is indicated accurately as infinity of space whose parts are connected each other as a unity at an open, unlimited space.

in spite of this one-dimension finite quantities is non-existence and is not parts of one quantitative continuum and only as an reference for comparing sizes in Axiom 3.

Theorem 3. It is known from theorem 2 that the except for finite length quantities of one-dimension the concept of gradually increases multidimensions is nonexistence (this multidimensions is different from infinite quantities of infinite dimensions of Axiom 3.) regardless of whether the dimensions in common sense (such as in Axiom 1 and Axiom 2) represents finite or infinite quantities. Taking an example, it is meaningless to talk about 1 dimension straight line that extends to infinite distance, because of even though considering it being infinite in the orientation of extending to infinite distance, it is finite in the other orientations in which axiom 3 is violated .Further, it is non-existence that any extensibility of space are made of combinations of finite quantities and infinite quantities since the parts of infinitely great must be connected to form a whole at an infinite distance to conform the definition of infinitely many quantities (all parts must be infinitely quantities) in Axiom 3. Seeing Figure 3. We can say that the space we see is just components of infinitely great. For the same reason, any line, or plane, or any outline of having properties of gradually increase dimension draw in space, such as circle, is meaningless ref. [3] [4] [5]. Seeing Figure 4. In additional, it does not make sense for us to do differential geometry on space. The space is an infinitely great unified continuum representing all orientations (the unified whole).

Corollary 1. To explain this point more clearly, let's give an example for comparison. In the modern mathematics, we think that the space we see is threedimensional. Given a three-dimensional object, it has extensive ductility. Taking 
1

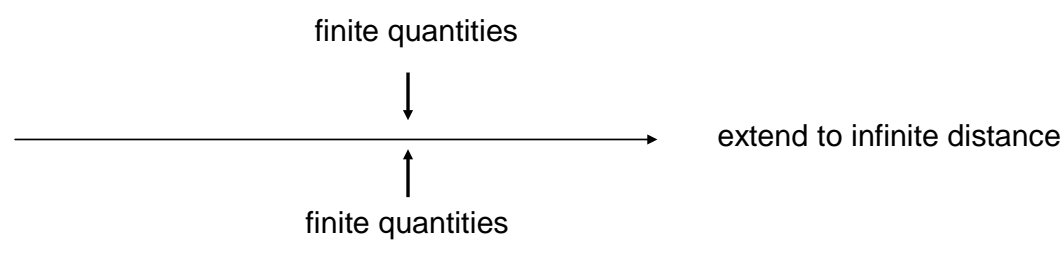

2

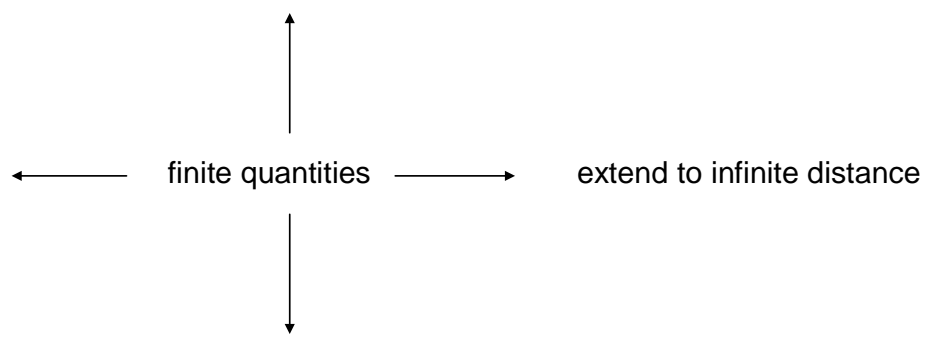

Figure 3. It is meaningless to talk about one-dimension straight line that extends to infinite distance (1), Further, It is non-existence that any extensibility of space are made of combinations of finite quantities and infinite quantities (2).
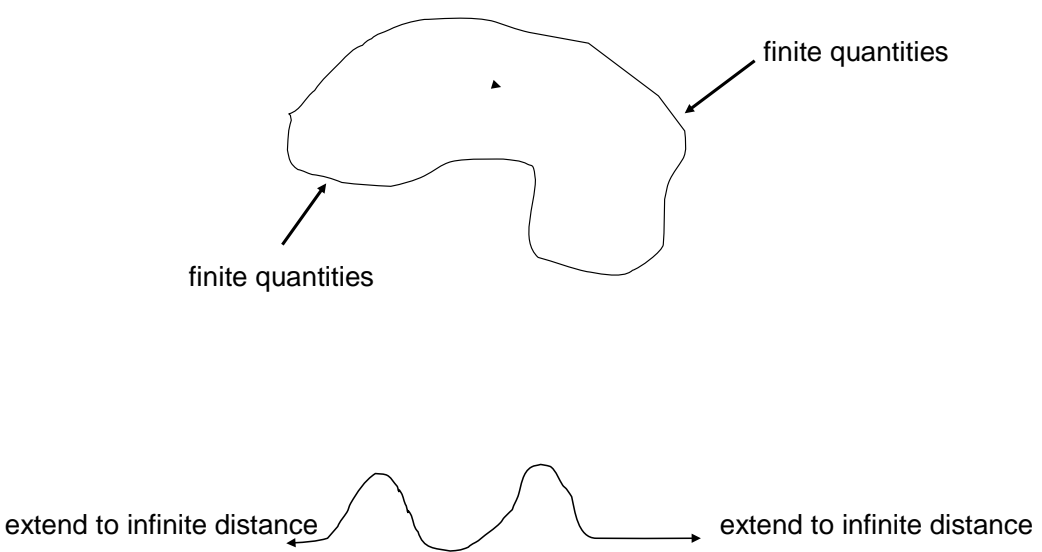

Figure 4. The any line, or plane, or any outline of having properties of gradually increase dimension draw in space is meaningless. Additional, it does not make sense for us to do differential geometry on space.

it as a reference, the space can extend to infinity. Here, the number and dimension value of the object can be compared, which can be gradually increased or decreased. Therefore, the geometry of the space can be seen as points, straight lines, curves, etc. For a given line segment with a certain length, it can be added, subtracted, multiplied and divided.

However, in Axiom 3, the above concepts need to be abandoned. In axiom 3 , there is only one unique quantity representing infinity. This infinity is the continuum we see. It can not be divided into smaller parts or added into larger parts. For example, it is meaningless to draw a one-dimensional curve in the continuum. This infinity is an infinite quantity with infinite dimension. Here, its quantity and dimension are unified into one concept, that is, the infinite quantity of infinite dimension. The operation of addition, subtraction, multiplication 


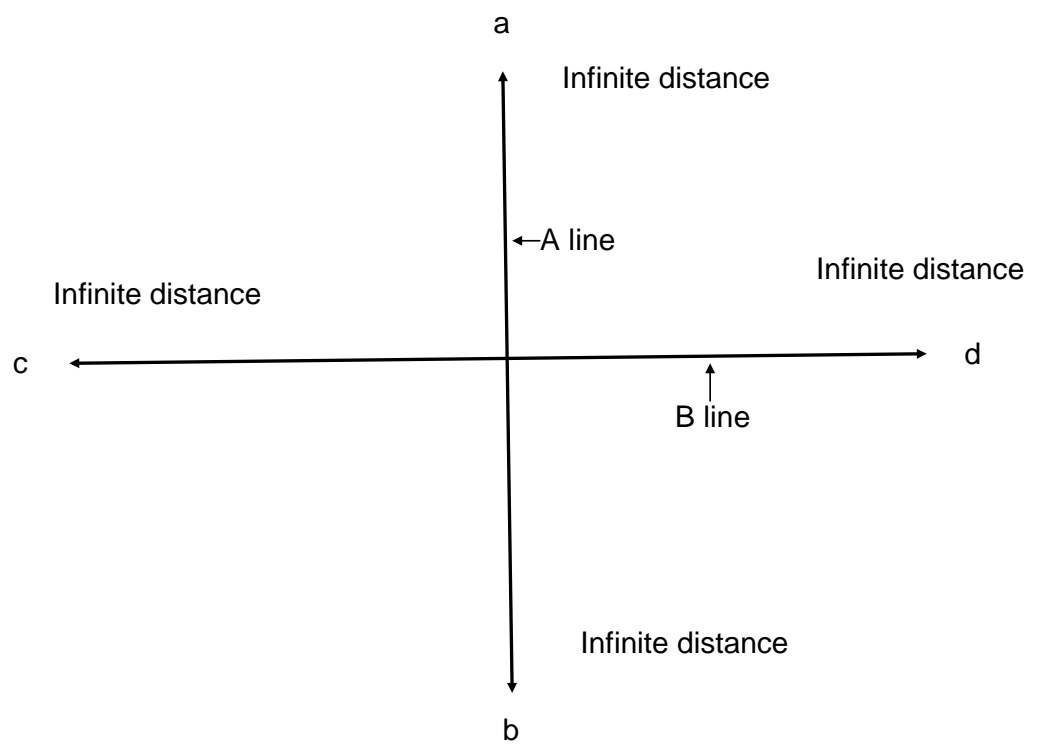

Figure 5. Line A and line B are straight lines perpendicular to each other. Point a and $\mathrm{b}$ is the points of line $\mathrm{A}$ at infinity. Point $\mathrm{c}$ and $\mathrm{d}$ is the points of line $\mathrm{B}$ at infinity. If the space is three-dimensional according to modern mathematics, then the four points of abcd are irrelevant. If the space is infinite dimensional as axiom 3 says, then the four points of abcd become one point at infinity, and the existence of line A and B line is becoming meaningless.

and division cannot be performed on this infinite quantity, and the operation of addition, subtraction, multiplication and division cannot be performed on this infinite dimension as well. Therefore, the space we see is not a three-dimensional body, but an infinite dimension. It is an infinite number (infinitely great quantity), which is connected into a unified whole in the infinite distance. Therefore, the concept of the azimuth direction of a certain line or surface here is meaningless, and all parts of the space we see are this one quantitative unity. As shown in Figure 5.

Theorem 4. It is known from theorem 2 that the one quantitative continuum indicating indefinitely great connected each other (actually only one quantity) as a unity at the infinite distance relative to any orientation (all orientations) of our existence. Any part of this one quantities unity is itself and any operations or algorithms building on this unity is itself. Considering the concept of time, it can be known from character 2 that if we take us as the origin dot and extend it to infinite distance, its end and beginning will become a quantity at infinite distance.

\section{Conclusion}

In this paper, The concept of $\mathrm{n}$ infinite dimensions has been proposed where the concept of 0 dimension, one-dimension, two-dimension, and three-dimension, the more high dimension, until infinite dimension, namely the concept of gradually increasings of dimension is non-existence and there is only a concept of infinitely great that is one quantitative continuum. The space we see is just these in- 
finitely great quantities implied by the change of direction. The accurate description of this one quantitative continuum is that its parts are connected each other as a unity at the infinite distance (infinitely great) relative to any orientation (all orientations) of our existence. It is unity in which its random parts are these infinitely great quantities and thus we call this unity as infinite quantities of infinite dimensions.

\section{Prospective}

So far, the exact value of any length of space and meaning of dimension of space have been described mathematically. So some readers ask what is the relationship between space and time? Taking example, how are some physical quantities such as velocity, acceleration, mass defined in axiom 3 ? We will describe properties of these quantities in detail in the later papers.

\section{Data Availability Statement}

The [DATA TYPE] data used to support the findings of this study are included within the article.

\section{Ethics Statement}

The study is informed consent for all authors in this manuscript.

\section{Funding}

This research received no specific grant from any funding agency in the public, commercial, or not-for-profit sectors.

\section{Author's Contributions}

Qing Li does full work in this manuscript.

\section{Conflicts of Interest}

The author declares no conflict of interest in preparing this article.

\section{References}

[1] Li, Q. (2021) A Geomerty Consisting of Singularities Containing Only Integers. (Preprint Research Square) https://doi.org/10.21203/rs.3.rs-219046/v1

[2] Li, Q. (2020) The Meaning of the Infinitely Great. (Preprint Authorea) https://doi.org/10.22541/au.160822935.50569408/v1

[3] Culler, M. and Shalen, P.B. (1983) Varieties of Group Representations and Splittings of 3-Manifolds. Annals of Mathematics, 117, 109-146. https://doi.org/10.2307/2006973

[4] Davis, M.W. (1983) Groups Generated by Reflections and Aspherical Manifolds Not Covered by Euclidean Space. Annals of Mathematics, 117, 293-327. https://doi.org/10.2307/2007079

[5] Ruelle, D. (1982) Characteristic Exponents and Invariant Manifolds in Hilbert Space. Annals of Mathematics, 115, 243-290. https://doi.org/10.2307/1971392 